\title{
Bohr-Sommerfeld Lagrangian submanifolds as minima of convex functions
}

\author{
Alexandre Vérine
}

March 21, 2018

\begin{abstract}
We prove that every closed Bohr-Sommerfeld Lagrangian submanifold $Q$ of a symplectic/Kähler manifold $X$ can be realised as a Morse-Bott minimum for some 'convex' exhausting function defined in the complement of a symplectic/complex hyperplane section $Y$. In the Kähler case, 'convex' means strictly plurisubharmonic while, in the symplectic case, it refers to the existence of a Liouville pseudogradient. In particular, $Q \subset X \backslash Y$ is a regular Lagrangian submanifold in the sense of Eliashberg-Ganatra-Lazarev.
\end{abstract}

Rational convexity properties of Lagrangian submanifolds were first discovered in $\mathbf{C}^{2}$ by Duval, who later investigated them further with his collaborators and students. In particular, generalising a result established by Duval-Sibony [DS95] in $\mathbf{C}^{n}$, Guedj Gue99 obtained the following theorem: in a complex projective manifold $X$, every closed Lagrangian submanifold $Q$ is rationally convex, which means that $X \backslash Q$ is filled up with smooth complex hypersurfaces. More precisely, these complex hypersurfaces $Y$ are very ample divisors of arbitrarily large degrees, so their complements are affine manifolds and possess exhausting $\mathbf{C}$-convex 1 functions $f: X \backslash Y \rightarrow \mathbf{R}$. In this work, which was motivated by the study of vanishing cycles in global Picard-Lefschetz theory, we give a necessary and sufficient condition for the existence of such a function $f$ admitting $Q$ as a Morse-Bott (i.e. transversally non-degenerate) minimum. This condition refers to a Kähler class and can be more generally stated as follows in the symplectic setting:

Definition 1. Let $(X, \omega)$ be an integral symplectic manifold, meaning that $X$ is a closed manifold and $\omega$ a symplectic form with integral periods. We say that a Lagrangian submanifold $Q$ satisfies the Bohr-Sommerfeld condition - or simply is Bohr-Sommerfeld - if the homomorphism $\mathrm{H}_{2}(X, Q, \mathbf{Z}) \rightarrow \mathbf{R}$ defined by integration of $\omega$ takes its values in $\mathbf{Z}$.

\footnotetext{
${ }^{1}$ Here the term $\mathbf{C}$-convex is used as a substitute for "strictly plurisubharmonic".
} 
In the Kähler setting, our main result is:

Theorem 2. Let $(X, \omega)$ be a closed integral Kähler manifold and $Q$ a closed Lagrangian submanifold satisfying the Bohr-Sommerfeld condition. Then, for every sufficiently large integer $k$, there exist a complex hyperplane section $Y$ of degree $k$ in $X$ avoiding $Q$ and an exhausting $\mathbf{C}$-convex function $f: X \backslash Y \rightarrow \mathbf{R}$ that has a Morse-Bott minimum at $Q$ and is Morse away from $Q$ with finitely many critical points.

To be more explicit, there exists a holomorphic line bundle $L \rightarrow X$ with first Chern class $\omega$ such that the complex hypersurface $Y$ is the zero-set of a holomorphic section of the $k$-th tensor power $L^{k}$ of $L$.

In AGM01, Auroux-Gayet-Mohsen reproved Guedj's above theorem and extended it to the symplectic setting using the ideas and techniques developed by Donaldson in [Don96]. Theorem 2 also has a symplectic version, whose statement below appeals to the following terminology:

- A symplectic hyperplane section of degree $k$ in a closed integral symplectic manifold $(X, \omega)$ is a symplectic submanifold $Y$ of codimension 2 that is Poincaré dual to $k \omega$.

- A function $f: X \backslash Y \rightarrow \mathbf{R}$ is $\omega$-convex if it admits a pseudogradient that is a Liouville (i.e. $\omega$-dual to some primitive of $\omega$ ) vector field .

With this wording, Donaldson's main theorem in [Don96] is that every closed integral symplectic manifold contains symplectic hyperplane sections of all sufficiently large degrees. Furthermore, according to Auroux-Gayet-Mohsen [AGM01, such symplectic hyperplane sections can be constructed away from any given closed Lagrangian submanifold. On the other hand, Giroux showed in Gir18 that, for all sufficiently large degrees, the complements of Donaldson's symplectic hyperplane sections admit exhausting $\omega$-convex functions (and hence are Weinstein manifolds). Mixing these ingredients, we obtain:

Theorem 3. Let $(X, \omega)$ be a closed integral symplectic manifold and $Q$ a closed Bohr-Sommerfeld Lagragian submanifold of $X$. Then, for every sufficiently large integer $k$, there exist a symplectic hyperplane section $Y$ of degree $k$ in $X$ avoiding $Q$ and an exhausting $\omega$-convex function $f: X \backslash Y \rightarrow \mathbf{R}$ that has a Morse-Bott minimum at $Q$ and is Morse away from $Q$ with finitely many critical points.

In EGL15, Eliashberg-Ganatra-Lazarev introduced the following definition: a Lagrangian submanifold $Q$ in a Weinstein manifold $(W, \omega)$ is 'regular' if there exists a Liouville pseudogradient on $W$ that is tangent to $Q$ (or equivalently there exists a primitive of $\omega$ vanishing on $Q$ ). This property, which implies that $Q$ is 
an exact Lagrangian submanifold, is known for quite a long time to be a strong constraint. For instance, it is elementary to see (without any holomorphic curve theory) that a closed Lagrangian submanifold in $\mathbf{C}^{n}$ cannot be regular. In the same time, though we do not have any example of a non-regular closed exact Lagrangian submanifold in a Weinstein manifold, we do not know any general method to prove that exact Lagrangian submanifolds should a priori be regular. Theorems 2 and 3 show that, in the complement of the complex and symplectic hyperplane sections constructed, the Bohr-Sommerfeld Lagrangian submanifold $Q$ is included in the zero-set of a Liouville pseudogradient and is therefore regular.

In section 1 we explain why the Bohr-Sommerfeld condition is necessary for our purposes and describe some of properties of Bohr-Sommerfeld Lagrangians. In section 2 we prove theorem [3, applying the main technical result from Gir18. In section 3 we prove theorem 2 and a complex-geometric analogue, using techniques that go back to [DS95].

Acknowledgements. This work is part of my Ph.D. prepared at ÉNS de Lyon under the supervision of Emmanuel Giroux. I warmly thank him for his help and support and Jean-Paul Mohsen for his comments on a draft of this paper. This work was supported by the LABEX MILYON (ANR-10-LABX-0070) of Université de Lyon, within the program "Investissements d'Avenir" (ANR-11-IDEX-0007) operated by the French National Research Agency (ANR), and by the UMI 3457 of CNRS-CRM.

\section{Bohr-Sommerfeld Lagrangian submanifolds...}

Let us first remark that Cieliebak-Mohnke proved, in [CM17, Theorem 8.3], a version of the main theorem of [AGM01 that is specific to Bohr-Sommerfeld Lagrangian submanifolds.

The Bohr-Sommerfeld condition in theorems 2 and 3 is necessary, indeed:

Lemma 4. Let $(X, \omega)$ be a closed symplectic manifold and $Q$ a Lagrangian submanifold. Suppose that there exist a symplectic hyperplane section $Y \subset X$ of degree $k$ avoiding $Q$ and $\lambda$ a primitive of $\omega$ over $X \backslash Y$ such that $\left.\lambda\right|_{Q}$ is exact. Then $Q$ is a Bohr-Sommerfeld Lagrangian submanifold of $(X, k \omega)$.

Proof. It suffices to prove the following (well-known) claim: Let $X$ be a closed connected oriented manifold, $Y \subset X$ a closed codimension 2 submanifold and $\omega$ a non-exact closed 2-form on $X$ that is Poincaré-dual to $Y$. Then, for every compact surface $\Sigma \subset X$ with boundary disjoint from $Y$ and primitive $\lambda$ of $\omega$ on $X \backslash Y$ such that $\left.\lambda\right|_{Q}$ is exact,

$$
\int_{\Sigma} \omega=\Sigma \cdot Y
$$


We first suppose that $Y$ is connected. For any embedded 2-disc $D$ intersecting $Y$ transversely at one point, with sign $\epsilon(D)= \pm 1$, set $r:=\epsilon(D)\left(\int_{D} \omega-\int_{\partial D} \lambda\right)$. The 'residue' $r$ does not depend on the disc $D$. Indeed, for two such discs $D$ and $D^{\prime}$, connectedness of $Y$ gives an oriented cylinder $C$ in $X \backslash Y$ bounding $-\epsilon\left(D^{\prime}\right) \partial D^{\prime}$ and $\epsilon(D) \partial D$ so by Stokes theorem,

$$
\epsilon\left(D^{\prime}\right) \int_{\partial D^{\prime}} \lambda-\epsilon(D) \int_{\partial D} \lambda=\int_{C} \omega=\epsilon\left(D^{\prime}\right) \int_{D^{\prime}} \omega-\epsilon(D) \int_{D} \omega .
$$

Let $\Sigma \subset X$ be a compact surface intersecting $Y$ away from $\partial \Sigma$. By a general position argument we may suppose the intersection is transverse. For each point $p_{i} \in \Sigma \cap Y$, take a disc $D_{i} \subset \Sigma$ that intersects $Y$ only at $p_{i}$. Stokes theorem gives $\int_{\Sigma \backslash \cup_{i} D_{i}} \omega=-\sum_{i} \int_{\partial D_{i}} \lambda$, then:

$$
\int_{\Sigma} \omega=\Sigma . Y r
$$

Since $\omega$ is not exact, we can apply (11) to some closed surface $\Sigma_{0}$ with $\Sigma_{0} \cdot Y=\int_{\Sigma_{0}} \omega$ $\neq 0$. This gives $r=1$; so (1) proves the claim.

Suppose $Y$ is not connected. If $\operatorname{dim} X \geq 3$, the cycle $[Y]$ may be represented by a closed connected submanifold, namely an embedded (away from $\partial \Sigma_{0}$ ) connected sum of the connected components of $Y$. If $\operatorname{dim} X=2$, we may represent $[Y]$ by some integral multiple of any point. Consequently, we reduce to the previous case.

Meanwhile, the Bohr-Sommerfeld condition can be easily obtained after a modification of the symplectic form:

Lemma 5 (Approximation and rescaling). Let $(X, \omega)$ be a closed symplectic manifold and $Q$ a closed Lagrangian submanifold. Then there exists a small closed 2-form $\epsilon$ and an integer $k$ such that $Q$ is a Bohr-Sommerfeld Lagrangian submanifold of $(X, k(\omega+\epsilon))$.

Proof. We argue as in AGM01: the 2 -form $\omega$ vanishes on $Q$ so, in view of the exact sequence $\cdots \rightarrow H^{2}(X, Q ; \mathbf{R}) \rightarrow H^{2}(X ; \mathbf{R}) \rightarrow H^{2}(Q ; \mathbf{R}) \rightarrow \cdots$, it is the image of a relative class $c \in H^{2}(X, Q ; \mathbf{R})$. We approximate $c$ by some $r \in H^{2}(X, Q ; \mathbf{Q})$ and take a small closed form $\epsilon$ vanishing on $Q$ that represents $c-r$. Then the closed form $\omega-\epsilon$ is symplectic, vanishes on $Q$ and its relative periods - given by evaluation of $r$ - are rational.

We now give the characterisation of Bohr-Sommerfeld Lagrangian submanifolds that we will use to prove theorems 2 and 3 . 
Lemma 6 (Hermitian flat line bundles). Let $(X, \omega)$ be an integral symplectic manifold and $Q$ a submanifold. Then $Q$ is a Bohr-Sommerfeld Lagrangian submanifold if and only if there exist a Hermitian line bundle $L \rightarrow X$ and a unitary connection $\nabla$ of curvature $-2 i \pi \omega$ such that $\left.(L, \nabla)\right|_{Q}$ is a trivial flat bundle. If $Q$ is a BohrSommerfeld Lagrangian and, in addition, $(X, \omega)$ is Kähler, then one can take for $(L, \nabla)$ a holomorphic Hermitian line bundle with its Chern connection.

Proof. Suppose that $Q$ is a Bohr-Sommerfeld Lagrangian submanifold. Since $\omega$ has integral periods, we may fix a lift $c$ of its cohomology class to $\mathrm{H}^{2}(X, \mathbf{Z})$. We take a Hermitian line bundle $L_{0} \rightarrow X$ with first Chern class $c$ and a unitary connection $\nabla_{0}$ of curvature $-2 i \pi \omega$. The submanifold $Q$ is Lagrangian so the restriction $\left.\left(L_{0}, \nabla_{0}\right)\right|_{Q}$ is a flat Hermitian bundle.

We will construct a flat Hermitian line bundle $\left(L_{1}, \nabla_{1}\right) \rightarrow X$ whose restriction to $Q$ is isomorphic to $\left.\left(L_{0}, \nabla_{0}\right)\right|_{Q}$. Then the desired line bundle will be $L_{0} \otimes L_{1}^{-1}$.

Recall that flat Hermitian line bundles over a manifold $Y$ are classified up to isomorphism by their holonomy representation $\mathrm{H}_{1}(Y, \mathbf{Z}) \rightarrow \mathrm{U}(1)$ (cf. proposition 3.6.15 in [Thu97]). To construct the flat bundle $\left(L_{1}, \nabla_{1}\right)$ it suffices to extend the holonomy representation $\rho: \mathrm{H}_{1}(Q, \mathbf{Z}) \rightarrow \mathrm{U}(1)$ of the flat bundle $\left.\left(L_{0}, \nabla_{0}\right)\right|_{Q}$ to a homomorphism $\mathrm{H}_{1}(X, \mathbf{Z}) \rightarrow \mathrm{U}(1)$.

We first show that $\rho$ vanishes on the kernel of the group homomorphism $i: \mathrm{H}_{1}(Q, \mathbf{Z}) \rightarrow \mathrm{H}_{1}(X, \mathbf{Z})$ induced by inclusion. Consider the exact sequence of the pair $(X, Q)$ :

$$
\cdots \rightarrow \mathrm{H}_{2}(X, Q ; \mathbf{Z}) \stackrel{\partial}{\rightarrow} \mathrm{H}_{1}(Q, \mathbf{Z}) \stackrel{i}{\rightarrow} \mathrm{H}_{1}(X, \mathbf{Z}) \rightarrow \cdots
$$

where $\partial$ is the homomorphism given by the boundary of chains. It suffices to show that $\rho \circ \partial=0$. Every $a \in \mathrm{H}_{2}(X, Q ; \mathbf{Z})$ can be represented by an embedded surface $\Sigma \subset X$ whose (possibly empty) boundary is included in $Q$. It then follows from (well-known) lemma 7 that:

$$
\rho(\partial a)=\exp \left(2 i \pi \int_{a} \omega\right) .
$$

Since the Lagrangian submanifold $Q$ is Bohr-Sommerfeld, $\rho(\partial a)=0$.

Thus $\rho$ factors through a homomorphism $\tilde{\rho}: \mathrm{H}_{1}(Q, \mathbf{Z}) /$ ker $i \rightarrow \mathrm{U}(1)$ where $\mathrm{H}_{1}(Q, \mathbf{Z}) /$ ker $i$ injects into $\mathrm{H}_{1}(X, \mathbf{Z})$. Now $\mathrm{U}(1)$ is a divisible abelian group so it is an injective Z-module (see for instance [Wei95, Corollary 2.3.2]). Hence $\tilde{\rho}$ extends to $H_{1}(X, \mathbf{Z})$.

In the case where $(X, \omega)$ is Kähler, the above Hermitian line bundle $\left(L_{0}, \nabla_{0}\right)$ can be chosen holomorphic with its Chern connection (see, e.g., Dem12, Theorem 13.9.b]). On the other hand the flat line bundle $\left(L_{1}, \nabla_{1}\right)$ is isomorphic to the quotient of the trivial flat bundle $\tilde{X} \times \mathbf{C}$ by the diagonal action of the fundamental group, acting on its universal cover $\tilde{X}$ by deck transformations and on 
C by the holonomy representation $\mathrm{H}_{1}(X, \mathbf{Z}) \rightarrow \mathrm{U}(1)$ (cf. proposition 3.6 .15 in [Thu97]). Therefore the trivial holomorphic structure and the trivial connection on $\tilde{X} \times \mathbf{C}$ respectively induce a holomorphic structure and the Chern connection on $L_{1}$. Consequently, the bundle $L_{0} \otimes L_{1}^{-1}$ has the desired properties.

Conversely, let $(X, \omega)$ be a symplectic manifold and a Hermitian line bundle $L \rightarrow X$ with a unitary connexion of curvature $-2 i \pi \omega$ such that $\left.(L, \nabla)\right|_{Q}$ is a trivial flat bundle. Then the (trivial) holonomy representation $\rho$ of $\left.(L, \nabla)\right|_{Q}$ satisfies (2); so $Q$ is a Bohr-Sommerfeld Lagrangian.

Lemma 7 (Gauss-Bonnet). Let $X$ be a manifold and $L \rightarrow X$ a Hermitian line bundle with a unitary connection $\nabla$ whose curvature 2 -form is written $-2 i \pi \omega$. Let $\Sigma$ be a connected oriented surface with non-empty boundary and $f: \Sigma \rightarrow X$ a map. The holonomy of $\nabla$ along the loop $\left.f\right|_{\partial \Sigma}$ is $\exp \left(2 i \pi \int_{\Sigma} f^{\star} \omega\right) \in \mathrm{U}(1)$.

Proof. We may assume $X=\Sigma$ and $f=\operatorname{Id}_{\Sigma}$ by pulling back the line bundle $L$ by $f$. There is a unit section $s: \Sigma \rightarrow L$. In the trivialisation of $L$ given by $s$ there is a primitive $\alpha$ of $\omega$ such that the connection $\nabla$ reads $d-2 i \pi \alpha$. By Stokes theorem

$$
\int_{\Sigma} \omega=\int_{\partial \Sigma} \alpha
$$

We may assume that $\partial \Sigma$ is connected. Take $\beta:[0,1] \rightarrow \partial \Sigma$ a parametrisation of $\partial \Sigma$. For every unit parallel lift $\gamma:[0,1] \rightarrow L$ of $\beta$ and for all $t \in[0,1]$, $\gamma^{\prime}(t)=2 i \pi \gamma(t)\left(\beta^{\star} \alpha\right)_{t}\left(\partial_{t}\right)$ hence

$$
2 i \pi \int_{\partial \Sigma} \alpha=\int_{[0,1]} \frac{\gamma^{\prime}(t)}{\gamma(t)} d t=\log \frac{\gamma(1)}{\gamma(0)} .
$$

An exponentiation gives the result.

\section{$2 \quad$...As minima of $\omega$-convex functions}

In this section we prove theorem 3 so $Q$ is a closed Bohr-Sommerfeld Lagrangian submanifold in a closed integral symplectic manifold $(X, \omega)$. We fix an $\omega$-compatible almost complex structure $J$ on $X$ and denote by $g=\omega(\cdot, J \cdot)$ the corresponding Riemannian metric. We denote by $\lambda_{0}$ the Liouville form on $T^{\star} Q$. The metric induced by $g$ on $Q$ determines a norm $|\cdot|$ on each fibre of $T^{\star} Q \rightarrow Q$. We define the function $f_{0}: T^{\star} Q \rightarrow \mathbf{R}_{\geq 0}$ by $f_{0}(p):=\pi|p|^{2}$. Using Weinstein's normal form theorem, we identify a neighbourhood $N$ of $Q \subset(X, \omega)$ with a tube $\left\{f_{0}<c\right\}$ around the zero section $Q$ in $\left(T^{\star} Q, \mathrm{~d} \lambda_{0}\right)$ in such a way that, for all $q \in Q$, the subspaces $T_{q} Q, T_{q}^{\star} Q \subset T_{q}\left(T^{\star} Q\right)$ are $g$-orthogonal.

Using Lemma 6, we fix a Hermitian line bundle $L \rightarrow X$ with a unitary connection $\nabla$ of curvature $-2 \pi i \omega$ and a unit parallel section $s_{0}$ of the flat bundle 
$\left.(L, \nabla)\right|_{Q}$. Parallel transport by $\nabla$ along the rays in the fibres of $T^{\star} Q$ extends $s_{0}$ to a section $s:\left.N \rightarrow L\right|_{N}$. We extend the section $s_{0}$ by $s_{0}:=e^{-f_{0}} s:\left.N \rightarrow L\right|_{N}$.

In the following, we denote by $L^{k}$ the $k$-th tensor power of the line bundle $L$, the induced connection has curvature $-2 k \pi i \omega$. We set $g_{k}:=k g$ the rescaled metric. For any integer $r \geq 0$, we endow the vector bundle $\otimes^{r} T^{\star} X \otimes L^{k}$ with the connection induced by the Levi-Civita connection for the metric $g_{k}$ and our connection on $L^{k}$; we still write this connection $\nabla$. The $J$-linear and $-J$-linear parts of the connexion $\nabla$ are written $\nabla^{\prime}$ and $\nabla^{\prime \prime}$. We define the $\mathcal{C}^{r}$ norm of a section $u: X \rightarrow L^{k}$ by $\|u\|_{\mathcal{C}^{r}, g_{k}}:=\sup |u|+\sup |\nabla u|_{g_{k}}+\cdots+\sup \left|\nabla^{r} u\right|_{g_{k}}$.

For any 1 -form $\lambda$ on $X$, we will denote by $\vec{\lambda}$ the vector field that is $k \omega$-dual to $\lambda$, where $k$ will be given by the context.

Lemma 8. There exists a constant $C>0$ such that, for every integer $k \geq 1$, the function $f_{0}$ and the section $s_{0}^{k}$ satisfy the following bounds on $N$ :

$$
\begin{gathered}
\overrightarrow{\lambda_{0}} \cdot\left(k f_{0}\right) \geq C^{-1}\left(\left|\overrightarrow{\lambda_{0}}\right|_{g_{k}}^{2}+\left|\mathrm{d}\left(k f_{0}\right)\right|_{g_{k}}^{2}\right), C^{-1}\left(k f_{0}\right)^{1 / 2} \leq\left|\mathrm{d}\left(k f_{0}\right)\right|_{g_{k}} \leq C\left(k f_{0}\right)^{1 / 2} \\
\left|\nabla s_{0}^{k}\right|_{g_{k}} \leq C\left(k f_{0}\right)^{1 / 2} e^{-k f_{0}},\left\|\nabla^{2} s_{0}^{k}\right\|_{\mathcal{C}^{0}, g_{k}} \leq C \text { and }\left\|\nabla^{\prime \prime} s_{0}^{k}\right\|_{\mathcal{C}^{1}, g_{k}} \leq C k^{-1 / 2}
\end{gathered}
$$

Proof. By rescaling, it suffices to establish the first two bounds of the statement for $k=1$. The function $f_{0}$ is Lyapounov for the vector field $\overrightarrow{\lambda_{0}}$. This implies the first bound. The submanifold $Q$ is a Morse-Bott minimum for $f_{0}$, hence the second bound.

Since $s_{0}=e^{-f_{0}} s$ with $s$ parallel,

$$
\nabla s_{0}=-\mathrm{d} f_{0} e^{-f_{0}} s+e^{-f_{0}} \nabla s=-\left(\mathrm{d} f_{0}+2 \pi i \lambda_{0}\right) s_{0} .
$$

Therefore, $\nabla s_{0}$ vanishes identically on the zero section. Hence, there exists a constant $C>0$ such that $\left|\nabla s_{0}\right|_{g} \leq C f_{0}^{1 / 2}$. Moreover, the 1 -jet of $\nabla^{\prime \prime} s_{0}$ vanishes identically on $Q$. Indeed, by the identity $\lambda_{0}=-\omega\left(\cdot, \overrightarrow{\lambda_{0}}\right)$ (here $k=1$ ) and by $J$-linearity of the 1 -form $g\left(\cdot, \overrightarrow{\lambda_{0}}\right)-i \omega\left(\cdot, \overrightarrow{\lambda_{0}}\right)$,

$$
\left.\nabla^{\prime \prime} s_{0}=-2 \pi\left(\frac{\mathrm{d} f_{0}}{2 \pi}+i \lambda_{0}\right)\right)^{\prime \prime} s_{0}=-2 \pi\left(\frac{\mathrm{d} f_{0}}{2 \pi}-g\left(\cdot, \overrightarrow{\lambda_{0}}\right)\right)^{\prime \prime} s_{0}
$$

so it suffices to show that the 1 -jet of the 1 -form $\frac{\mathrm{d} f_{0}}{2 \pi}-g\left(\cdot, \overrightarrow{\lambda_{0}}\right)$ vanishes identically along $Q$. Its 0 -jet clearly vanishes, and, for each vector $v=\left(v_{1}, v_{2}\right)$ in the $g$ orthogonal sum $\left.T\left(T^{\star} Q\right)\right|_{Q}=T Q \oplus T^{\star} Q$,

$$
\mathrm{d}\left(g\left(\cdot, \overrightarrow{\lambda_{0}}\right)\right)(v, v)=g\left(v, v \cdot \overrightarrow{\lambda_{0}}\right)=g\left(v, v_{2}\right)=g\left(v_{2}, v_{2}\right)=\left(\mathrm{d}^{2} f_{0}\right)(v, v) /(2 \pi),
$$

hence its 1-jet vanishes too. Consequently, there exists a constant $C>0$ such that $\left|\nabla \nabla^{\prime \prime} s_{0}\right|_{g} \leq C f_{0}^{1 / 2}$ and $\left|\nabla^{\prime \prime} s_{0}\right|_{g} \leq C f_{0}$. Therefore, by the Leibniz rule, we obtain 
the desired bounds on $\nabla s_{0}^{k}$ and $\nabla^{2} s_{0}^{k}$, and the two bounds $\left|\nabla^{\prime \prime} s_{0}^{k}\right|_{g_{k}} \leq C k^{1 / 2} f_{0} e^{-k f_{0}}$, $\left|\nabla \nabla^{\prime \prime} s_{0}^{k}\right|_{g_{k}} \leq\left(k f_{0}^{3 / 2}+f_{0}^{1 / 2}\right) C e^{-k f_{0}}$. The two latter real-valued Gaussian functions of $f_{0}$ both reach their global maximum at Constant $\times k^{-1}$ so we obtain the last bound of the statement.

We will state next lemma using Auroux's following

Definition 9. Sections $s_{k}: X \rightarrow L^{k}$ are called asymptotically holomorphic if there exists a constant $C>0$ such that for every positive integer $k,\left\|\nabla^{\prime \prime} s_{k}\right\|_{\mathcal{C}^{1}, g_{k}} \leq C k^{-1 / 2}$ and $\left\|s_{k}\right\|_{\mathcal{C}^{2}, g_{k}} \leq C$.

The following result was already observed in Auroux-Gayet-Mohsen AGM01, Remark p.746]. Recall that our neighbourhood $N$ of $Q$ is identified with the cotangent tube $\left\{f_{0}<c\right\}$.

Lemma 10. Let $\beta: N \rightarrow[0,1]$ be a compactly supported function (independent of k) with $\beta=1$ on a tube $\left\{f_{0}<b\right\}$. Then, the sections $s_{0, k}:=\beta s_{0}^{k}: X \rightarrow L^{k}$ are asymptotically holomorphic.

Proof of lemma 10. The sections $s_{0}^{k}$ satisfy the estimates of lemma 8 on $N$. Then, there exists a constant $C>0$ such that:

$$
\begin{aligned}
\left\|\nabla^{\prime \prime} s_{0, k}\right\|_{\mathcal{C}^{0}, g_{k}} & \leq\|d \beta\|_{\mathcal{C}^{0}, g_{k}} \sup _{\left\{f_{0}>b\right\}}\left|s_{0}^{k}\right|+\left\|\nabla^{\prime \prime} s_{0}^{k}\right\|_{\mathcal{C}^{0}, g_{k}} \\
& \leq C k^{1 / 2} e^{-b k}+C k^{-1 / 2} .
\end{aligned}
$$

Similarly:

$$
\begin{aligned}
\left\|\nabla \nabla^{\prime \prime} s_{0, k}\right\|_{\mathcal{C}^{0}, g_{k}} & \leq\left\|d^{2} \beta\right\|_{\mathcal{C}^{0}, g_{k}} \sup _{\left\{f_{0}>b\right\}}\left|s_{0}^{k}\right|+2\|d \beta\|_{\mathcal{C}^{0}, g_{k}} \sup _{\left\{f_{0}>b\right\}}\left|\nabla s_{0}^{k}\right|_{g_{k}}+\left\|\nabla \nabla^{\prime \prime} s_{0}^{k}\right\|_{\mathcal{C}^{0}, g_{k}} \\
& \leq C k e^{-b k}+2 C k^{1 / 2}\left(C k^{1 / 2} c^{1 / 2} e^{-b k}\right)+C k^{-1 / 2} .
\end{aligned}
$$

Hence, there exists a constant $C>0$ such that, for all $k,\left\|\nabla^{\prime \prime} s_{0, k}\right\|_{\mathcal{C}^{1}, g_{k}} \leq C k^{-1 / 2}$. In the same way, we obtain the bound $\left\|s_{0, k}\right\|_{\mathcal{C}^{2}, g_{k}} \leq C$.

Giroux's theorem below provides transverse perturbations our sections $s_{0, k}$ with the following property

Definition 11 (Giroux). Let $\kappa \in(0,1)$. A section $s: X \rightarrow L^{k}$ is called $\kappa$ quasiholomorphic if $\left|\nabla^{\prime \prime} s\right| \leq \kappa\left|\nabla^{\prime} s\right|$ at each point of $X$.

Theorem 12 ([Gir18, Proposition 13]). Let $\epsilon>0, \kappa \in(0,1)$ and $s_{0, k}: X \rightarrow L^{k}$ asymptotically holomorphic sections. Then, for any sufficiently large integer $k$, there exists a section $s_{1, k}: X \rightarrow L^{k}$ with the following properties: 
- $s_{1, k}$ vanishes transversally;

- $s_{1, k}$ is $\kappa$-quasiholomorphic;

- $\left\|s_{1, k}-s_{0, k}\right\|_{\mathcal{C}^{1}, g_{k}}<\epsilon$;

- $-\log \left|s_{1, k}\right|:\{p \in X, s(p) \neq 0\} \rightarrow \mathbf{R}$ is a Morse function.

Let us now bring the arguments together to prove theorem 3 .

Proof of theorem 3. Using lemma 10, we fix sections $s_{0, k}: X \rightarrow L^{k}$ with $s_{0, k}=s_{0}^{k}$ on a tube $\left\{f_{0}<b\right\}$. We then fix $\epsilon \in(0,1)$ and take sections $s_{1, k}: X \rightarrow L^{k}$ provided by theorem [12, The subset $Y:=\left\{s_{1, k}=0\right\} \subset(X, \omega)$ is a symplectic hyperplane section of degree $k$ (because of the first two properties of theorem 12 , see for instance proposition 3 in Don96]) avoiding the submanifold $Q$ (because $\left|s_{0}\right|=1$ on $Q$ and by the third property of theorem 12).

It remains to construct an $\omega$-convex exhaustion $f: X \backslash Y \rightarrow \mathbf{R}$ that has a Morse-Bott minimum at $Q$ and is Morse away from $Q$ with finitely many critical points. On the one hand, the function $f_{0, k}:=k f_{0}: N \rightarrow \mathbf{R}$ has a Morse-Bott minimum at $Q$ and, by Lemma 8 , is Lyapounov for the Liouville vector field $\overrightarrow{\lambda_{0}}$ with a uniform Lyapounov constant in the metric $g_{k}$. On the other hand, the exhaustive function $f_{1, k}:=-\log \left|s_{1, k}\right|$ is Morse (by the last property of theorem 12) and has finitely many critical points (because $s_{1, k}$ vanishes transversally). A pseudgradient for $f_{1, k}$ is provided by Giroux's following lemma. Before stating it, we set $\lambda_{1, k}$ the real 1-form such that, in the unitary trivialisation of $\left.L^{k}\right|_{X \backslash Y}$ given by $s_{1, k} /\left|s_{1, k}\right|$, the connection $\nabla$ reads $\mathrm{d}-2 k \pi i \lambda_{1, k}$. We also recall that the notation $\vec{\lambda}$ stands for the $k \omega$-dual vector field to a given 1 -form $\lambda$, where $k$ is given by the context.

Lemma 13 ([Gir18, Lemma 12]). Let $\kappa \in(0,1)$ and $s_{1, k}: X \rightarrow L^{k} a \kappa$ quasiholomorphic section. Then

$$
\overrightarrow{\lambda_{1, k}} \cdot f_{1, k} \geq \frac{1}{2} \frac{1-\kappa^{2}}{1+\kappa^{2}}\left(\left|\mathrm{~d} f_{1, k}\right|_{g_{k}}^{2}+\left|\overrightarrow{\lambda_{1, k}}\right|_{g_{k}}^{2}\right)
$$

Hence the function $f_{1, k}$ is Lyapounov for the Liouville vector field $\overrightarrow{\lambda_{1, k}}$, with a uniform Lyapounov constant in the metric $g_{k}$. Finally, the desired function $f$ is constructed in the following lemma, by gluing, on an annular region $\left\{a<f_{0, k}<b\right\}$ about $Q$, the standard (Morse-Bott) Weinstein structure $\left(\overrightarrow{\lambda_{0}}, f_{0, k}\right)$ on $T^{\star} Q$ with the Weinstein structure $\left(\overrightarrow{\lambda_{1, k}}, f_{1, k}\right)$ given by Giroux's above theorem.

Lemma 14. Let $\kappa \in(0,1)$ and $a, b \in(0, c)$ with $a<b$. Then, for every sufficiently small $\epsilon \in(0,1)$ and for every $k \geq k_{0}(\epsilon)$ sufficiently large, there exist a Liouville 
vector field $\vec{\lambda}$ on $X \backslash Y$ and a Lyapounov function $f: X \backslash Y \rightarrow \mathbf{R}$ for $\vec{\lambda}$ such that $(\vec{\lambda}, f)=\left(\overrightarrow{\lambda_{0}}, f_{0, k}\right)$ on $\left\{f_{0, k} \leq a\right\}$ and $(\vec{\lambda}, f)=\left(\overrightarrow{\lambda_{1, k}}, f_{1, k}\right)$ away from $\left\{f_{1, k}<b\right\}$.

Proof. We will omit the indices $k$ in the proof. We observe first that, for sufficiently small $\epsilon$, the section $s_{1}$ does not vanish on $N$ and the tube $\left\{f_{1}<b\right\}$ is contained in $N$ (because $\left\|s_{1}-s_{0}\right\|_{\mathcal{C}^{0}}<\epsilon$ and $\inf _{\left\{f_{0}<c\right\}}\left|s_{0}\right|>e^{-c}$ ).

Let us prove that there exists a constant $C>0$ (independent of $k, \epsilon$ ) such that

$$
\left\|f_{0}-f_{1}\right\|_{\mathcal{C}^{1}(N), g_{k}} \leq C \epsilon
$$

and that, for sufficiently small $\epsilon>0$, the form $\lambda_{1}-\lambda_{0}$ is exact on $N$. On the one hand, $f_{0}-f_{1}=\operatorname{Re} \log \left(s_{1} s_{0}^{-1}\right)$ and, since $u_{j}:=s_{j} /\left|s_{j}\right|$ satisfies $\nabla u_{j}=-2 k \pi i \lambda_{j} u_{j}$,

$$
\lambda_{1}-\lambda_{0}=\frac{1}{2 k \pi i}\left(u_{0}^{-1} \nabla u_{0}-u_{1}^{-1} \nabla u_{1}\right)=\frac{1}{2 k \pi i} \mathrm{~d} \log \left(u_{0} u_{1}^{-1}\right)=\frac{1}{2 k \pi} \mathrm{d} \arg \left(s_{1} s_{0}^{-1}\right) .
$$

On the other hand, $\left\|\log \left(s_{1} s_{0}^{-1}\right)\right\|_{\mathcal{C}^{1}, g_{k}} \leq C \epsilon$; this is a consequence of the three bounds $\left\|s_{1}-s_{0}\right\|_{\mathcal{C}^{1}, g_{k}}<\epsilon$, inf $\left|s_{0}\right|>e^{-c}$, and $\left\|\nabla s_{0}\right\|_{\mathcal{C}^{0}, g_{k}} \leq$ Constant (from lemma 8). In particular we obtain the bound (3) and, for $\epsilon$ sufficiently small, $\left\|\arg \left(s_{1} s_{0}^{-1}\right)\right\|_{\mathcal{C}^{0}}<\pi / 3$ so $\lambda_{1}-\lambda_{0}$ is exact.

Now, for $\epsilon<(b-a) / 2$, the annular region $\left\{a<f_{0}<b\right\}$ contains the level set $\left\{f_{1}=(b-a) / 2\right\}$ (by the bound (3) $)$. We construct a Lyapounov function $f: X \rightarrow \mathbf{R}$ for the vector field $\overrightarrow{\lambda_{0}}$ with $f=f_{0}$ on $\left\{f_{0} \leq a\right\}$ and $f=f_{1}$ away from $\left\{f_{1}<(b-a) / 2\right\}$. It suffices to show that $\overrightarrow{\lambda_{0}}$ is transverse to the level sets of $f_{0}$ and $f_{1}$ in the region $\left\{a<f_{0}, f_{1}<(b-a) / 2\right\}$; indeed, increasing from $a$ to $b$ along each trajectory of $\overrightarrow{\lambda_{0}}$ gives a function $f$ transverse to the level sets of $f_{0}$ and $f_{1}$. There exists a constant $C^{\prime}>0$ such that $\left\|\mathrm{d} f_{0}\right\|_{g_{k}} \geq C^{\prime}$ (by lemma 8). By the latter bound and (3), $\left\|\mathrm{d} f_{1}\right\|_{g_{k}} \geq C^{\prime}-C \epsilon$. So, by the Lyapounov conditions, there exists a constant $C^{\prime}>0$ such that $\overrightarrow{\lambda_{0}} \cdot f_{0} \geq C^{\prime}$ and $\overrightarrow{\lambda_{1}} \cdot f_{1} \geq C^{\prime}$. By the latter bound and again (3), $\overrightarrow{\lambda_{0}}$ is transverse to the level sets of $f_{1}$.

It remains to construct a Liouville vector field $\vec{\lambda}$ transverse to the level sets of the function $f$ in the annular region $\left\{(b-a) / 2<f_{1}<b\right\}$ (where $f=f_{1}$ ), and coïnciding with $\overrightarrow{\lambda_{0}}$ on $\left\{f_{1}<(b-a) / 2\right\}$ and with $\overrightarrow{\lambda_{1}}$ outside $\left\{f_{1} \leq b\right\}$. In view of our initial computation, we have a function $H$ such that $\overrightarrow{\lambda_{1}}-\overrightarrow{\lambda_{0}}=\overrightarrow{\mathrm{d} H}$. Let us fix a cutoff function $\beta: \mathbf{R} \rightarrow[0,1]$ such that $\beta=0$ near $\mathbf{R}_{\leq(b-a) / 2}$ and $\beta=1$ near $\mathbf{R}_{\geq b}$ and set $\beta_{1}:=\beta \circ f_{1}$. Then the vector field $\vec{\lambda}:=\overrightarrow{\lambda_{0}}+\overrightarrow{\mathrm{d}\left(\beta_{1} H\right)}$ is Liouville and satisfies the desired boundary conditions. Moreover, $\overrightarrow{\beta_{1}}$ is tangent to the level sets of $f_{1}$ and, by the above paragraph, $\overrightarrow{\lambda_{0}}$ and $\overrightarrow{\lambda_{1}}$ are transverse to these, so $\vec{\lambda}=\left(1-\beta_{1}\right) \overrightarrow{\lambda_{0}}+\beta_{1} \overrightarrow{\lambda_{1}}+\overrightarrow{\beta_{1}} H$ is transverse to these too. 
Remark 15 (An alternative proof of the regularity of $Q \subset X \backslash Y$ ). For sufficiently large $k$, it is possible to choose our $\kappa$-quasiholomorphic perturbation $s_{1, k}: X \rightarrow L^{k}$ (vanishing transversally and away from $Q$ ) of $s_{0, k}$ in such a way that the quotient function $\left.\left(s_{1, k} / s_{0, k}\right)\right|_{Q}$ is real-valued. The latter property, which can be achieved by implementing techniques from Auroux-Munoz-Presas' AMP05] in the proof of [Gir18, Proposition 13], implies that the Liouville pseudogradient $\overrightarrow{\lambda_{1, k}}$ of the function $-\log \left|s_{1, k}\right|$ is tangent to $Q$.

\section{3 ...As minima of C-convex functions}

This section deals with the proof of Theorem 2, so $Q$ is a closed BohrSommerfeld Lagrangian submanifold in a closed integral Kähler manifold $(X, \omega)$. Using Lemma 6, we fix a holomorphic Hermitian line bundle $L \rightarrow X$ with Chern curvature $-2 \pi i \omega$ and a parallel unit section $s_{0}:\left.Q \rightarrow L\right|_{Q}$. We denote by $\nabla$ the Chern connection. We denote by $d_{k}$ the distance function to $Q$ in the metric $g_{k}=k \omega(\cdot, i \cdot)$ and we set $B_{k}(Q, c):=\left\{d_{k}<c\right\}$. We recall that we endow the vector bundle $\otimes^{r} T^{\star} X \otimes L^{k}$ with the connection induced by the Levi-Civita connection for the metric $g_{k}$ and the connection on $L^{k}$ - we still write this connection $\nabla$. We define the $\mathcal{C}^{r}$ norm of a section $u: X \rightarrow L^{k}$ by $\|u\|_{\mathcal{C}^{r}, g_{k}}:=\sup |u|+\sum_{j=1}^{r} \sup \left|\nabla^{j} u\right|_{g_{k}}$.

Since $Q$ is a totally real submanifold of $X$, it has a neighbourhood on which the squared distance function $d_{1}^{2}$ is $\mathbf{C}$-convex (see for instance Proposition 2.15 in [CE12]), so some neighbourhood $N:=B_{1}(Q, c)$ is a Stein manifold. By results of Oka [Oka39] and Grauert Gra58], the line bundle $\left.L\right|_{N}$ admits a non-vanishing holomorphic section $s:\left.N \rightarrow L\right|_{N}$. Furthermore, given any integer $r \geq 1$, CE12, Proposition 5.55] shows that the complex-valued function $s_{0} /\left(\left.s\right|_{Q}\right)$ extends to a smooth function $F: N \rightarrow \mathbf{C}$ such that the form $\mathrm{d}^{\prime \prime} F$ vanishes identically along $Q$ together with its $r$-jet. We will eventually choose $r=n$, the complex dimension of the manifold $X$. We then extend $s_{0}$ over $N$ by setting $s_{0}:=F s:\left.N \rightarrow L\right|_{N}$.

Remark 16 (The real-analytic case). If the submanifold $Q$ is real-analytic, then one can take for $s_{0}:\left.N \rightarrow L\right|_{N}$ a holomorphic section. Indeed, one may ensure that the connection $\nabla$ on the bundle $L$ provided by Lemma 6 is real-analytic. In that case, the section $s_{0}:\left.Q \rightarrow L\right|_{Q}$ is real-analytic and can be complexified.

Lemma 17. There exist a constant $C>0$ such that, for every integer $k \geq 1$, the section $s_{0}^{k}:\left.N \rightarrow L^{k}\right|_{N}$ satisfies the following bounds on $N$ :

$$
\begin{aligned}
\left|2 \pi k \omega-\mathrm{dd}^{c} \log \right| s_{0}^{k}||_{g_{k}} & \leq C k^{-1 / 2} d_{k}, \\
e^{-C d_{k}^{2}} \leq\left|s_{0}^{k}\right| & \leq e^{-d_{k}^{2} / C} \\
\left|\nabla s_{0}^{k}\right|_{g_{k}} \leq C d_{k} e^{-d_{k}^{2} / C},\left\|\nabla^{\prime \prime} s_{0}^{k}\right\|_{\mathcal{C}^{1}, g_{k}} & \leq C k^{-r / 2}, k^{-r / 2} .
\end{aligned}
$$


Proof. We first observe that $\nabla s_{0}$ vanishes at every point $p \in Q$. Indeed, $T_{p} X$ $=T_{p} Q \oplus i T_{p} Q$ (because $Q$ is totally real of middle dimension), $\nabla s_{0}(p)=\nabla^{\prime} s_{0}(p)$ (because $\nabla^{\prime \prime} s_{0}(p)=0$ ) and $\nabla s_{0}(p)$ vanishes on $T_{p} Q$ (because $\left.s_{0}\right|_{Q}$ is parallel). Thus, there exists a constant $C>0$ such that $\left|\nabla s_{0}\right| \leq C d_{1}$. Similarly, since the $r$-jet of $\nabla^{\prime \prime} s_{0}$ vanishes identically on $Q$, there exists a constant $C>0$ such that $\left|\nabla^{\prime \prime} s_{0}\right|_{g_{1}} \leq C d_{1}^{r+1}$ and $\left|\nabla \nabla^{\prime \prime} s_{0}\right|_{g_{1}} \leq C d_{1}^{r}$.

We now consider the function $f_{0}:=-\log \left|s_{0}\right|$. Clearly, $f_{0}(p)=0$ and

$$
\mathrm{d} f_{0}(p)=\frac{1}{2} \mathrm{~d} \log \left(\left|s_{0}\right|^{2}\right)=\frac{1}{2}\left|s_{0}\right|^{-2} \mathrm{~d}\left(\left|s_{0}\right|^{2}\right)=\left|s_{0}\right|^{-2} \operatorname{Re}\left\langle\nabla s_{0}, s_{0}\right\rangle=0 .
$$

Moreover,

$$
2 \pi \omega_{p}+\left(\mathrm{dd}^{c} f_{0}\right)_{p}=\mathrm{dd}^{c} \log \left|\frac{s}{s_{0}}\right|=-i \mathrm{~d}^{\prime} \mathrm{d}^{\prime \prime} \log \left|\frac{s}{s_{0}}\right|^{2}=-\left(i \mathrm{~d}^{\prime} \mathrm{d}^{\prime \prime} \log |F|^{2}\right)_{p}=0
$$

because the 1-jet of the form $\mathrm{d}^{\prime \prime} F$ vanishes at $p$. Therefore, there exists a constant $C>0$ such that $\left|2 \pi \omega+\mathrm{dd}^{c} f_{0}\right|_{g} \leq C d_{1}$. Multiplicating this by $k$ gives the first bound of the statement. On the other hand, the Hessian quadratic form $\left(\mathrm{d}^{2} f_{0}\right)_{p}: T_{p} X \rightarrow \mathbf{R}$ vanishes on $T_{p} Q$ and satisfies, for every vector $v \in T_{p} X$,

$$
\left(\mathrm{d}^{2} f_{0}\right)(v, v)+\left(\mathrm{d}^{2} f_{0}\right)(i v, i v)=-\left(\mathrm{dd}^{c} f_{0}\right)(v, i v)=2 \pi \omega(v, i v)=2 \pi g(v, v) .
$$

Hence, $\left(\mathrm{d}^{2} f_{0}\right)_{p}$ is positive definite on $i T_{p} Q$ and $Q$ is a Morse-Bott minimum for $f_{0}$. Since $Q$ is compact, one can find a constant $C>0$ such that, on some neighbourhood of $Q$ for the metric $g_{1}$ :

$$
d_{1}^{2} / C \leq f_{0} \leq C d_{1}^{2}
$$

In other words, $e^{-C d_{1}^{2}} \leq|s| \leq e^{-d_{1}^{2} / C}$. We obtain the second bound of the statement by taking the $k$-th power. The third bound and the bounds

$$
\left|\nabla^{\prime \prime} s_{0}^{k}\right|_{g} \leq C k d_{1}^{r+1} e^{-k d_{1}^{2} / C}, \quad\left|\nabla \nabla^{\prime \prime} s_{0}^{k}\right|_{g} \leq C k d_{1}^{r}\left(1+k d_{1}^{2}\right) e^{-k d_{1}^{2} / C}
$$

follow from this bound and the bounds on $\nabla s_{0}, \nabla^{\prime \prime} s_{0}$ and $\nabla \nabla^{\prime \prime} s_{0}$ by the Leibniz rule applied to $s_{0}^{k}$. The two latter real-valued Gaussian functions of $d_{1}$ both reach their global maximum at Constant $\times k^{-1 / 2}$. By expressing these bounds in the rescaled metric $g_{k}$, we obtain the last bound of the statement.

The following is the main result of this section.

Proposition 18. Let $\rho \in(0, c)$. There exist holomorphic sections $s_{k}: X \rightarrow L^{k}$ such that, for every $\epsilon>0$ and for $k \geq k_{0}(\epsilon)$ sufficiently large, $s_{k}$ vanishes transversally and $\left\|s_{k}-s_{0}^{k}\right\|_{\mathcal{C}^{1}, g_{k}}<\epsilon$ on $B_{1}(Q, \rho)$, the $\rho$-neighbourhood of $Q$ in the metric $g$. 
We postpone the proof of proposition 18 and first explain how it implies theorem 2 ,

Proof of theorem 2. We fix a radius $\rho \in(0, c)$ and, by proposition 18, holomorphic sections $s_{k}: X \rightarrow L^{k}$ : for every $\epsilon>0$ and for $k \geq k_{1}(\epsilon)$ sufficiently large, the zeroset $Y:=s_{k}^{-1}(0)$ is a (smooth) complex hyperplane section and $\left\|s_{k}-s_{0}^{k}\right\|_{\mathcal{C}^{1}, g_{k}}<\epsilon$ on $B_{1}(Q, \rho)$. By the second and third inequalities in lemma 17, there exists a constant $C>0$ (independent of $k$ and $\epsilon$ ) such that, for $\epsilon>0$ sufficiently small, on $B_{k}(Q, \rho)$, the functions $f_{1}:=-\log \left|s_{k}\right|$ and $f_{0}:=-\log \left|s_{0}^{k}\right|$ satisfy

$$
\left\|f_{1}-f_{0}\right\|_{\mathcal{C}^{1}, g_{k}}<C \epsilon \text {. }
$$

Take a cutoff function $\beta_{k}: X \rightarrow[0,1]$ supported in $B_{k}(Q, \rho)$, with $\beta_{k}=1$ on $B_{k}(Q, \rho / 2)$ and $\left\|\beta_{k}\right\|_{\mathcal{C}^{2}, g_{k}} \leq C^{\prime}$ for some constant $C^{\prime}>0$ (independent of $k$ ). The function $f:=\beta_{k} f_{0}+\left(1-\beta_{k}\right) f_{1}: X \backslash Y \rightarrow \mathbf{R}$ is exhausting, reaches a Morse-Bott minimum at $Q$ and its critical points remain in a compact subset. (We remark that, for sufficiently small $\epsilon$, this minimum is global. Indeed, on $\left\{\beta_{k}=1\right\}, f=f_{0}$, and on $\left\{\beta_{k}<1\right\}, f_{1} \geq-\log \left(\left|s_{0}\right|+\epsilon\right) \geq-\log \left(e^{-\rho^{2} / C}+\epsilon\right)>0$. $)$

Let us show that $f$ is $\mathbf{C}$-convex. First, since $s_{k}$ is holomorphic, $-\mathrm{dd}^{c} f_{1}=2 k \pi \omega$. Then, by the first bound of lemma 17, there exists a constant $C^{\prime \prime}>0$ such that $\left\|\operatorname{dd}^{c}\left(f_{0}-f_{1}\right)\right\|_{\mathcal{C}^{0}, g_{k}} \leq C^{\prime \prime} k^{-1 / 2}$. Hence,

$$
\begin{aligned}
& \left\|2 k \pi \omega+\mathrm{dd}^{c} f\right\|_{\mathcal{C}^{0}, g_{k}} \\
& =\left\|\mathrm{dd}^{c}\left(\beta_{k}\left(f_{0}-f_{1}\right)\right)\right\|_{\mathcal{C}^{0}, g_{k}} \\
& \leq\left\|\beta_{k}\right\| C^{\prime \prime} k^{-1 / 2}+\left\|\left(f_{0}-f_{1}\right) \mathrm{dd}^{c} \beta_{k}\right\|+\left\|\mathrm{d}\left(f_{0}-f_{1}\right) \wedge \mathrm{d}^{c} \beta_{k}\right\|+\left\|\mathrm{d}^{c}\left(f_{1}-f_{0}\right) \wedge \mathrm{d} \beta_{k}\right\| \\
& \leq C^{\prime \prime} k^{-1 / 2}+3(C \epsilon) C^{\prime} .
\end{aligned}
$$

Consequently, for every $\epsilon>0$ sufficiently small and for every $k \geq k_{0}(\epsilon)$ sufficiently large, $\left\|2 k \pi \omega+\mathrm{dd}^{c} f\right\|_{\mathcal{C}^{0}, g_{k}}<2 \pi$. This inequality ensures that the function $f$ is C-convex. A $\mathcal{C}^{2}$-small perturbation of the function $f$ with support in a compact subset of $Y \backslash Q$ is Morse away from $Q$ and satisfies the properties of theorem 2 .

Our next aim is to prove proposition [18, In the following lemma the $\mathrm{L}^{2}$-norm of a section $s: X \rightarrow \otimes^{r} T^{\star} X \otimes L^{k}$ for the rescaled metric $g_{k}$ is defined by

$$
\|s\|_{\mathrm{L}^{2}, g_{k}}:=\left(\int_{X}|s|_{g_{k}}^{2} \frac{(k \omega)^{n}}{n !}\right)^{1 / 2} .
$$

Lemma 19. Let $\beta: X \rightarrow[0,1]$ a function supported in $N$ with $\beta=1$ on a tube $B(Q, \rho)$. There exists a constant $C>0$ such that the sections $s_{0, k}:=\beta s_{0}^{k}: X \rightarrow L^{k}$ satisfy the following bounds:

$$
\left\|\nabla^{\prime \prime} s_{0, k}\right\|_{\mathcal{C}^{1}, g_{k}} \leq C k^{-r / 2},\left\|\nabla^{\prime \prime} s_{0, k}\right\|_{\mathrm{L}^{2}, g_{k}} \leq C k^{(n-r) / 2}
$$


Proof. The sections $s_{0}^{k}$ satisfy the bounds of Lemma 17 on $N$. Then, there exists a constant $C>0$ such that:

$$
\begin{aligned}
\left\|\nabla^{\prime \prime} s_{0, k}\right\|_{\mathcal{C}^{0}, g} & \leq\|\mathrm{d} \beta\|_{\mathcal{C}^{0}, g} \sup _{\left\{d_{1}>\rho\right\}}\left|s_{0}^{k}\right|+\sup _{B(Q, 2 \rho)}\left|\nabla^{\prime \prime} s_{0}^{k}\right|_{g} \\
& \leq C\left(e^{-k / C}+k^{-(r-1) / 2}\right)
\end{aligned}
$$

In the same way:

$$
\begin{aligned}
& \left\|\nabla \nabla^{\prime \prime} s_{0, k}\right\|_{\mathcal{C}^{0}, g} \\
& \leq\left\|\mathrm{d}^{2} \beta\right\|_{\mathcal{C}^{0}, g} \sup _{\left\{d_{1}>\rho\right\}}\left|s_{0}^{k}\right|+2\|\mathrm{~d} \beta\|_{\mathcal{C}^{0}, g} \sup _{\left\{d_{1}>\rho\right\}}\left|\nabla s_{0}^{k}\right|_{g}+\sup _{B(Q, 2 \rho)}\left|\nabla \nabla^{\prime \prime} s_{0}^{k}\right|_{g} \\
& \leq C e^{-k / C}+C e^{-k / C}+C k^{-(r-2) / 2} .
\end{aligned}
$$

Since

$$
\left\|\nabla^{\prime \prime} s_{0, k}\right\|_{\mathrm{L}^{2}, g_{k}} \leq C k^{n / 2}\left\|\nabla^{\prime \prime} s_{0, k}\right\|_{\mathcal{C}^{0}, g_{k}}
$$

the $\mathcal{C}^{1}$ and the $\mathrm{L}^{2}$ norms, in the metric $g_{k}$, satisfy the bounds of the statement.

We now use the following version of Hörmander's $L^{2}$-estimates:

Theorem 20 (cf. [Dem12, theorem VIII.6.5] and the discussion thereafter). Let $(X, \omega)$ be a closed integral Kähler manifold and $L \rightarrow X$ a holomorphic Hermitian line bundle with Chern curvature $-2 \pi i \omega$. Set $C:=\sup \left|\frac{\operatorname{Ricci}(\omega)}{2 \pi}\right|_{g}$. Then, for every $k>C$ and for every smooth section $u: X \rightarrow \bigwedge^{1,0} T^{\star} X \otimes L^{k}$ such that $\nabla^{\prime \prime} u=0$, there exists a smooth section $t: X \rightarrow L^{k}$ satisfying:

$$
\nabla^{\prime \prime} t=u \text { and }\|t\|_{\mathrm{L}^{2}}^{2} \leq \frac{1}{n(k-C)}\|u\|_{\mathrm{L}^{2}}^{2} .
$$

Applying this theorem to the sections $s_{0, k}$ of lemma 19, we obtain smooth sections $t_{k}: X \rightarrow L^{k}$ satisfying $\left\|t_{k}\right\|_{L^{2}, g_{k}} \leq C k^{(n-r-1) / 2}$, and, for $k$ sufficiently large, $\nabla^{\prime \prime}\left(s_{0, k}-t_{k}\right)=0$. The following lemma converts our $\mathrm{L}^{2}$-estimates to $\mathcal{C}^{1}$ estimates.

Lemma 21. Let $(X, \omega)$ be a closed integral Kähler manifold, $L \rightarrow X$ a holomorphic Hermitian line bundle with Chern curvature $-2 \pi i \omega$. There exists a constant $C>0$ such that for every integer $k$ and for every section $t: X \rightarrow L^{k}$ :

$$
\|t\|_{\mathcal{C}^{1}, g_{k}} \leq C\left(\left\|\nabla^{\prime \prime} t\right\|_{\mathcal{C}^{1}, g_{k}}+\|t\|_{\mathrm{L}^{2}, g_{k}}\right) .
$$


Proof. The desired bound is local. At a given point $p \in X$, we will obtain it on a $g_{k^{-}}$ ball of uniform radius about $p$ - where, for sufficenlty large $k$, the geometry of $L^{k}$ compares with the trivial line bundle over the unit ball of euclidean space $\left(\mathbf{C}^{n}, g_{0}\right)$. There exist constants $r, C>0$ and a family (indexed by $p, k$ ) of holomorphic charts $\underline{z}_{p}^{k}: B_{k}(p, r) \rightarrow \mathbf{C}^{n}$ centered at $p$ such that,

$$
\left\|\left(\underline{z}_{p}^{k}\right)_{\star} g_{k}-g_{0}\right\|_{\mathcal{C}^{1}, g_{0}} \leq C k^{-1 / 2} \text { over }\left(\underline{z}_{p}^{k}\right)\left(B_{k}(p, r)\right) .
$$

Indeed, we take some constant $r>0$ and a smooth family (indexed by $p \in X$ ) of centered holomorphic charts $\underline{z}_{p}: B(p, r) \rightarrow \mathbf{C}^{n}$ with isometric differentials $\mathrm{d} \underline{z}_{p}(p)$ at the origin. The post-composition of $\underline{z}_{p}$ by the centered dilation $\mathbf{C}^{n} \rightarrow \mathbf{C}^{n}$ of ratio $k^{1 / 2}$ gives $\underline{z}_{p}^{k}$.

Let us take a Hörmander holomorphic peak section at $p$ (see for instance [Don96, Proposition 34]): for sufficiently large $k$, there exists a holomorphic section $s_{p}: X \rightarrow L^{k}$ satisfying the bounds:

$$
\left|s_{p}(p)\right|=1, \inf _{B_{k}(p, r)}\left|s_{p}\right| \geq C^{-1} \text { and }\left\|s_{p}\right\|_{\mathcal{C}^{1}, g_{k}} \leq C
$$

for some constant $C>0$ independent of $p$ and $k$.

Let $t$ be a section of $L^{k}$ and $p \in B\left(Q, \rho^{\prime}\right)$. We set $f:=\frac{t}{s_{p}}$. In view of the identities $\nabla t=\mathrm{d} f s_{p}+f \nabla s_{p}, \nabla \nabla t=\mathrm{d}^{2} f s_{p}+2 \mathrm{~d} f \otimes \nabla s_{p}+f \nabla \nabla s_{p}$, and the bounds on the peak sections, it suffices to show that for sufficently large $k$,

$$
\|f\|_{\mathcal{C}^{1}\left(B_{k}(p, r / 6)\right), g_{k}} \leq C\left\|\mathrm{~d}^{\prime \prime} f\right\|_{\mathcal{C}^{1}\left(B_{k}(p, r)\right), g_{k}}+C\|f\|_{\mathrm{L}^{2}\left(B_{k}(p, r)\right), g_{k}} .
$$

In the following, we will identify the domain of the chart $\underline{z}_{p}^{k}$ with its image in $\mathbf{C}^{n}$. We denote by $B_{0}(q, r)$ the ball of radius $r$ at a point $q$ in $\mathbf{C}^{n}$ and by $\mu$ the Euclidean volume form on $\mathbf{C}^{n}$. Let us prove the (standard) following bound:

$$
\|f\|_{\mathcal{C}^{1}\left(B_{0}(0, r / 5)\right), g_{0}} \leq C\left\|\mathrm{~d}^{\prime \prime} f\right\|_{\mathcal{C}^{1}\left(B_{0}(0, r / 2)\right), g_{0}}+C\|f\|_{\mathrm{L}^{2}\left(B_{0}(0, r / 2)\right), g_{0}} .
$$

This will end the proof because, in view of the comparaison (5) of the rescaled metric $g_{k}$ with the flat metric $g_{0}$, for sufficiently large $k$, we have the inclusions $B_{k}(p, r / 6) \subset B_{0}(0, r / 5)$ and $B_{0}(0, r / 2) \subset B_{k}(p, r)$, and there exists a constant $C>0$ (independent on $k$ and $p$ ) such that, over $B_{0}(0, r / 2)$,

$$
\mu \leq\left(1+C k^{-n / 2}\right) \frac{(k \omega)^{n}}{n !} \text { and }\left(1-C k^{-1 / 2}\right)|\cdot|_{g_{0}} \leq|\cdot| g_{g_{k}} \leq\left(1+C k^{-1 / 2}\right)|\cdot|_{g_{0}} .
$$

On the one hand, [HW68, Lemma 4.4] gives:

$$
\|f\|_{\mathcal{C}^{0}\left(B_{0}(0, r / 4)\right)} \leq C\left\|\mathrm{~d}^{\prime \prime} f\right\|_{\mathcal{C}^{0}\left(B_{0}(0, r / 2)\right)}+C\|f\|_{\mathrm{L}^{2}\left(B_{0}(0, r / 2)\right), g_{0}} .
$$


On the other hand, we have the following standard bound (cf. [CE12, Lemma 8.37] for instance):

$$
\|f\|_{\mathcal{C}^{1}\left(B_{0}(0, r / 5)\right), g_{0}} \leq C\left\|\mathrm{~d}^{\prime \prime} f\right\|_{\mathcal{C}^{1}\left(B_{0}(0, r / 4)\right), g_{0}}+C\|f\|_{\mathcal{C}^{0}\left(B_{0}(0, r / 4)\right)} .
$$

In the two above estimates the constants depend only on $r$ and $n$. Therefore we obtain the desired bound.

By lemma 21, we obtain the following estimate: for every $\epsilon>0$, for $k \geq k_{1}(\epsilon)$ sufficiently large,

$$
\left\|t_{k}\right\|_{\mathcal{C}^{1}, g_{k}} \leq C\left(\left\|\nabla^{\prime \prime} s_{0, k}\right\|_{\mathcal{C}^{1}, g_{k}}+k^{-1 / 2}\left\|s_{0, k}\right\|_{L^{2}, g_{k}}\right) \leq C k^{(n-r-1) / 2}<\epsilon / 2 .
$$

On the other hand, by Bertini theorem, for sufficiently large $k$ there exists a holomorphic section $s_{k}: X \rightarrow L^{k}$ vanishing transversally with

$$
\left\|s_{k}-\left(s_{0, k}-t_{k}\right)\right\|_{\mathcal{C}^{1}, g_{k}}<\epsilon / 2 .
$$

Therefore the sections $s_{k}$ satisfy the conclusions of proposition 18. This ends the proof of theorem 2 ,

Let us finish with a complex-geometric variant of theorem 2 ;

Theorem 22. Let $X$ be a closed complex manifold, a a Kähler class and $Q$ a closed submanifold. Suppose that $Q$ is a Bohr-Sommerfeld Lagrangian submanifold for some Kähler form in a. Then, there exists a holomorphic line budle $L \rightarrow X$ with first Chern class a, and, for every sufficiently large $k$, there exist a Hermitian metric $h_{k}$ on $L^{k}$ with positive Chern curvature and a holomorphic section $s_{k}: X \rightarrow L^{k}$ vanishing transversally such that the function $-\log \left|s_{k}\right|_{h_{k}}: X \backslash s_{k}^{-1}(0) \rightarrow \mathbf{R}$ has a Morse-Bott minimum at $Q$ and is Morse elsewhere.

Proof of theorem 22. We fix a Kähler form $\omega \in a$ with $\left.\omega\right|_{Q}=0$ as well as a Hermitian holomorphic line bundle $L \rightarrow X$ with Chern curvature $-2 i \pi \omega$ whose restriction to $Q$ is a trivial flat bundle (by lemma 6). We fix $\epsilon, \rho>0$ and repeat the construction of section 3 to obtain sections $s_{0}^{k}, s_{k}: X \rightarrow L^{k}$ with the properties stated in lemma 17 and proposition 18. We keep the notation $f_{0}=-\log \left|s_{0, k}\right|$ and $f_{1}=-\log \left|s_{k}\right|$.

To construct the desired Hermitian metric on $L^{k}$, we will proceed as in the final step of the proof of theorem 2 but we will modify the initial Hermitian metric $h^{k}$ of $L^{k}$ instead of the function $f_{1}$. Take a cutoff function $\beta_{k}: X \rightarrow[0,1]$ with support in $B_{k}(Q, \rho)$ with $\beta_{k}=1$ on $B_{k}(Q, \rho / 2)$ and such that $\left\|\mathrm{d} \beta_{k}\right\|_{\mathcal{C}^{1}, g_{k}}<C^{\prime}$, for some constant $C^{\prime}>0$ independent of $k$. We define a new Hermitian metric on $L^{k}$ by:

$$
h_{k}^{\prime}=e^{2 \beta_{k}\left(f_{1}-f_{0}\right)} h^{k} .
$$


The exhaustion function $-\log \left|s_{k}\right|_{h_{k}^{\prime}}:\left\{s_{k} \neq 0\right\} \rightarrow \mathbf{R}$ equals $f_{0}$ on $B_{k}(Q, \rho / 2)$ hence has a Morse-Bott local minimum at $Q$. Furthermore,

$$
2 k \pi \omega-\mathrm{dd}^{c} \log \left|s_{k}\right|_{h_{k}^{\prime}}=\mathrm{dd}^{c}\left(\beta_{k}\left(f_{1}-f_{0}\right)\right) .
$$

Therefore, by repeating the estimation (44), for every $\epsilon<\epsilon_{0}$ sufficiently small and for $k \geq k_{0}(\epsilon)$ sufficiently large, $\left\|2 k \pi \omega-\mathrm{dd}^{c} \log \left|s_{k}\right|_{h_{k}^{\prime}}\right\|_{\mathcal{C}^{0}, g_{k}}<2 \pi$. This inequality ensures that the function $-\log \left|s_{k}\right|_{h_{k}^{\prime}}$ is $\mathbf{C}$-convex. Finally, there exists a $\mathcal{C}^{2}$-small function $\eta_{k}: X \backslash Y \rightarrow \mathbf{R}$ with compact support away from $Q$ such that, setting the Hermitian metric $h_{k}^{\prime \prime}:=e^{-2 \eta_{k}} h_{k}^{\prime}$, the function $-\log \left|s_{k}\right|_{h_{k}^{\prime \prime}}=-\log \left|s_{k}\right|_{h^{\prime}}+\eta$ is Morse away from $Q$.

In conclusion, the Hermitian metric $h_{k}^{\prime \prime}$ and the sections $s_{k}: X \rightarrow L^{k}$ have the desired properties.

\section{References}

[AGM01] D. Auroux, D. Gayet, and J.-P. Mohsen. Symplectic hypersurfaces in the complement of an isotropic submanifold. Math. Ann. 321 (2001), 739-754.

[AMP05] D. Auroux, V. Muñoz, and F. Presas. Lagrangian submanifolds and Lefschetz pencils. J. Sympl. Geom. 3 (2005), 171-219.

[CM17] K. Cieliebak, K. Mohnke. Punctured holomorphic curves and Lagrangian embeddings. Invent. math. (2017) (https://doi.org/10.1007/ s00222-017-0767-8).

[CE12] K. Cieliebak and Y. Eliashberg. From Stein to Weinstein and BackSymplectic Geometry of Complex Affine Manifolds. Colloq. Publ. 59, Amer. Math. Soc., 2012.

[Dem12] J.-P. Demailly. Complex Analytic and Differential Geometry. Preprint 2012, Inst. Fourier, Grenoble (https://www-fourier.ujf-grenoble.fr/ demailly/man uscripts/agbook.pdf).

[Don96] S. K. Donaldson. Symplectic submanifolds and almost-complex geometry. J. Diff. Geom. 44 (1996), 666-705.

[DS95] J. Duval and N. Sibony. Polynomial convexity,rational convexity, and currents. Duke Math. J. 79 (1995) ,487-513. 
[EGL15] Y. Eliashberg, S. Ganatra, and O. Lazarev. Flexible Lagrangians. Preprint 2015 (arXiv.org/abs/1510.01287).

[Gir18] E. Giroux. Remarks on Donaldson's symplectic submanifolds. Preprint 2018 (arxiv.org/abs/1803.05929).

[Gra58] H. Grauert. Analytische Faserungen über holomorph-vollständigen Räumen. Math. Ann. 135 (1958), 263-273.

[Gue99] V. Guedj. Approximation of currents on complex manifolds. Math. Ann. 313 (1999), 437-474.

[Oka39] K. Oka. Sur les fonctions des plusieurs variables. III: Deuxième problème de Cousin. J. Sc. Hiroshima Univ. 9 (1939), 7-19.

[HW68] L. Hörmander and J. Wermer. Uniform approximation on compact sets in $\mathbf{C}^{n}$. Math. Scand. 223 (1968), 5-21.

[Thu97] W. P. Thurston. Three-Dimensional Geometry and Topology. Princeton Math. Ser. 35, S. Levy ed., Princeton Univ. Press, 1997.

[Wei95] C. A. Weibel. An Introduction to Homological Algebra. Cambridge Univ. Press, 1995. 